\section{天琴计划与国际合作}

胡一鸣 ${ }^{1,2}$, 梅健伟 ${ }^{1,2}$, 罗俊 $1,2^{*}$

1. 中山大学天琴中心, 珠海 519082;

2. 中山大学物理与天文学院, 珠海 519082

* 联系人, E-mail: junluo@mail.sysu.edu.cn

2016 年 2 月 11 日, LIGO(the Laser Interferometer Gravitational-Wave Observatory)科学合作组宣布于 2015 年 9 月 14 日探测到来自 GW150914 的引力波信号 ${ }^{[1]}$. 这是人 类首次直接观测到引力波, 也是人类首次观测到双黑洞并 合的天文现象. 全世界新闻媒体争相报道, 造成极大轰动 与回响. 引力波探测的实现使人类进人了利用引力波来探 测宇宙的全新时期 ${ }^{[2 \sim 6]}$.

引力波是爱因斯坦广义相对论的预言之一, 指黑洞、 中子星等极端致密天体的运动变化, 导致时空曲率产生扰 动, 然后以波动的形式由波源向外扩散传播的现象 ${ }^{[7]}$. 作 为一种天文观测的全新媒介, 引力波探测为人类研究宇宙 打开了全新窗口, 可以与传统电磁波观测手段形成互补, 进一步拓展我们对中子星、白矮星、黑洞等致密天体以及 星系和宇宙演化的认识 ${ }^{[8,9]}$. 除此之外, 引力波精密测量对 于揭示万有引力本质、检验引力理论也将发挥重大的引领 作用 ${ }^{[10,11]}$.

引力波同电磁波一样有广阔的频谱, 自然界中可探测 的引力波频率覆盖 $10^{3} \sim 10^{-16} \mathrm{~Hz}$ (宇宙年龄尺度)约 20 个量 级的跨度. 不同频段的引力波需要不同的探测手段, 引力 波的频率越低(波长越长), 所需要的探测器尺寸也越大. 为了探测频率在 $100 \mathrm{~Hz}$ 附近频段的引力波, LIGO 等地面 引力波探测器采用了千米尺度的激光干涉仪; 但是由于地 面振动、重力梯度噪声等影响, LIGO 对几赫兹以下的引力 波基本上没有探测能力. 在紧邻 LIGO 的毫赫兹 $\left(10^{-3} \mathrm{~Hz}\right)$ 频段, 分布着天文上极其重要的大质量黑洞等奇特的引力 波源. 为了打开毫赫兹频段的引力波探测窗口, 需要在太 空中构造十万到百万千米尺度的巨型激光干涉仪, 进行引 力波的空间探测.

相比于引力波的地面探测, 空间引力波探测从波源类 型、数量、信号强度、可探测的空间距离等各方面来说都 有明显的优势, 是开展引力波天文学研究不可替代的观测 手段 ${ }^{[9,12 ~ 16]}$. 开展空间引力波探测研究, 还可以极大推动 高精度时空参考体系构建、超长距离激光干涉测量、高精 度卫星平台等一大批尖端空间技术发展, 对于促进相关学 科发展、推动产业升级、服务国家重大战略需求等具有重 要的支撑作用和战略价值.

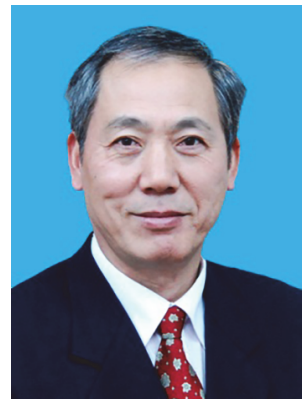

罗俊长江学者特聘教授、中国 科学院院士. 长期从事引力实验与 精密测量物理研究, 开展了牛顿万 有引力常数 $G$ 的精确测量, 实验结 果被国际科技数据委员会基本物理 常数任务组收录; 开展了光子静止 质量的实验检验, 实验结果被国际 粒子物理数据组收录; 开展了亚毫 米范围牛顿反平方定律的实验检验, 实验结果得到国际同行认可; 与他人合作开展了宏观旋转 物体等效原理的实验检验等基础科学研究.

20 世纪 70 年代末, 以陈嘉言为代表的科学家们在中 国开始了引力波探测的研究. 20 世纪 80 年代, 在华中科技 大学开始基础引力实验研究, 于 2000 和 2002 年开始布局 空间引力波探测的核心技术空间惯性传感和星间激光干 涉测量技术的研究. 此后, 与国内外同行一道, 积极酝酿 开展中国的空间引力波探测项目，包括在 2007 年的第 316 次香山科学会议上明确建议国家开展空间引力波探测研 究. 2013 年, 研制的静电悬浮加速度计成功进行了首次在 轨试验, 为我国自主开展空间引力波探测任务跨出关键的 第一步. 我们从 2013 年底开始讨论一个由中国主导的空 间引力波探测方案, 于 2014 年正式提出天琴计划, 并召开 第一届天琴空间科学任务研讨会. 在国内外专家多次研讨 的基础上, 于 2015 年完成并公布了天琴的初步概念方 案 ${ }^{[17]}$, 提出了在 21 世纪 30 年代实现空间引力波探测的任 务目标.

\section{1 天琴计划的提出}

\section{1 引力波探测及研究现状}

自从美国马里兰大学的 Weber ${ }^{[18]}$ 在 20 世纪 60 年代率 先开始利用被称为韦伯棒的探测器探测引力波后, 引力波 探测在国际上获得了广泛的关注. 目前, 从低频到高频的 各个频段都有不同的引力波探测器, 包括地面激光干涉 
仪、空间激光干涉仪、脉冲星计时阵列、宇宙微波背景辐 射探测等, 如图 1 所示. 各种探测方法主要针对的引力波 波源、引力波波频段以及科学问题各不相同, 它们在引力 波探测上相互补充, 无法相互取代 ${ }^{[8]}$.

地面激光干涉仪臂长通常在几千米级别(如美国的 LIGO 臂长为 $4 \mathrm{~km}^{[19]}$, 欧洲的 Virgo 臂长为 $3 \mathrm{~km}^{[20]}$, 日本 的 KAGRA(the Kamioka Gravitational Wave Detector)臂长 为 $3 \mathrm{~km}^{[21]}$ ). LIGO, Virgo 和 GEO600(臂长为 $600 \mathrm{~m}^{[22]}$ ) 都已 经运行多年，而 KAGRA 正在升级建设中，印度台站 LIGO-India 还在计划中 ${ }^{[23]}$. 目前, LIGO 和 Virgo 已经升级 成为 aLIGO(advanced LIGO) 和 AdV(advanced Virgo), 并且 还在不断提升其探测灵敏度. 地面探测主要针对百赫兹 $(100 \mathrm{~Hz})$ 附近频段的引力波.

脉冲星计时阵列探测引力波是指通过对地球周边一 系列脉冲星信号进行高精度测时记录, 以分析是否有引力 波信号的信息. 当前国际上主要的脉冲星计时阵列引力波 探测实验主要有澳大利亚的 PPTA(the Parkes Pulsar Timing Array) ${ }^{[24]}$, 欧洲的 EPTA(the European Pulsar Timing Array) ${ }^{[25]}$, 北美的 NANOGrav(North American Nanohertz Observatory for Gravitational Waves) ${ }^{[26]}$. 我国已经建成的 FAST(the Five-hundred-meter Aperture Spherical radio Telescope)也有望在未来的 PTA(Pulsar Timing Array)引力 波探测中发挥作用 ${ }^{[27]}$. 脉冲星计时阵列主要针对纳赫兹 $\left(10^{-9} \mathrm{~Hz}\right)$ 附近频段的引力波. 经过十多年的科学运行, 利 用这些实验数据已经可以对纳赫兹频段附近的引力波给 出有意义的限制.

宇宙微波背景辐射探测引力波是指通过对宇宙微波 背景辐射中光子的 $\mathrm{B}$ 模极化的高精度探测, 来获取有关原 初引力波的信息. 美国于 2001 年发射的 WMAP(Wilkinson Microwave Anisotrope Probe)卫星及欧洲于 2009 年发射的
Planck 卫星已经对原初引力波给出了很好的限制 ${ }^{[28,29]}$. 位 于南极的 BICEP(Background Imaging of Cosmic Extragalactic Polarization)望远镜于 2006 年开始运行, 并于 2016 年升级为 $\mathrm{BICEP} 3^{[30]}$. 我国正在进行的阿里计划也将通过 微波背景辐射观测来探测原初引力波 ${ }^{[31]}$. 宇宙微波背景辐 射探测主要针对 $10^{-16} \mathrm{~Hz}$ 附近频段的原初引力波.

\section{2 空间引力波探测}

毫赫兹频段附近的引力波频谱上，有着种类丰富的引 力波源：近有成千上万的银河系内双白矮星信号，远有宇 宙早期发生的大质量双黑洞并合; 既可以和地面引力波联 合开展多波段引力波测，研究恒星级致密双星，或者与电 磁观测等手段结合开展多信使天文学研究，也可以单打独 斗，分析极端质量比旋近等信号。对于上述波源，还可以 通过研究其互相交叠产生的随机引力波背景, 探究群体性 质. 这些引力波信号的探测和研究, 可以针对研究强场条 件下的引力本质和黑洞本质，恒星、星系和黑洞的形成、 演化和并合历史，以及宇宙的演化等关键科学问题，提供 无法通过其他方式获取的信息. 因此，开展毫赫兹频段的 引力波探测, 具有十分重要的科学意义 ${ }^{[9,11]}$.

地面激光干涉仪虽然可以用于百赫兹级别引力波信 号的观测, 但是在 $10 \mathrm{~Hz}$ 以下, 各类噪声接踵而至, 目前提 出的最先进实验方案都无法将灵敏频段延伸至 $1 \mathrm{~Hz}$ 以 下 $^{[32 ~ 35]}$. 在国际上, 经过长期讨论与实践, 基本形成共识: 开展毫赫兹频段引力波探测, 必须将探测器发射到太空中, 通过空间激光干涉实现 ${ }^{[36 ~ 40]}$

欧美国家从 20 世纪 80 年代提出空间引力波探测概念 方案 ${ }^{[36 ~ 40]}$. LISA(Laser Interferometer Space Antenna)于 20 世纪 90 年代初被提出 ${ }^{[41,42]}$, 并于 2015 年成功发射技术验 证星 LISA Pathfinder ${ }^{[43 \sim 5]}$. 在当前提出的空间引力波探测

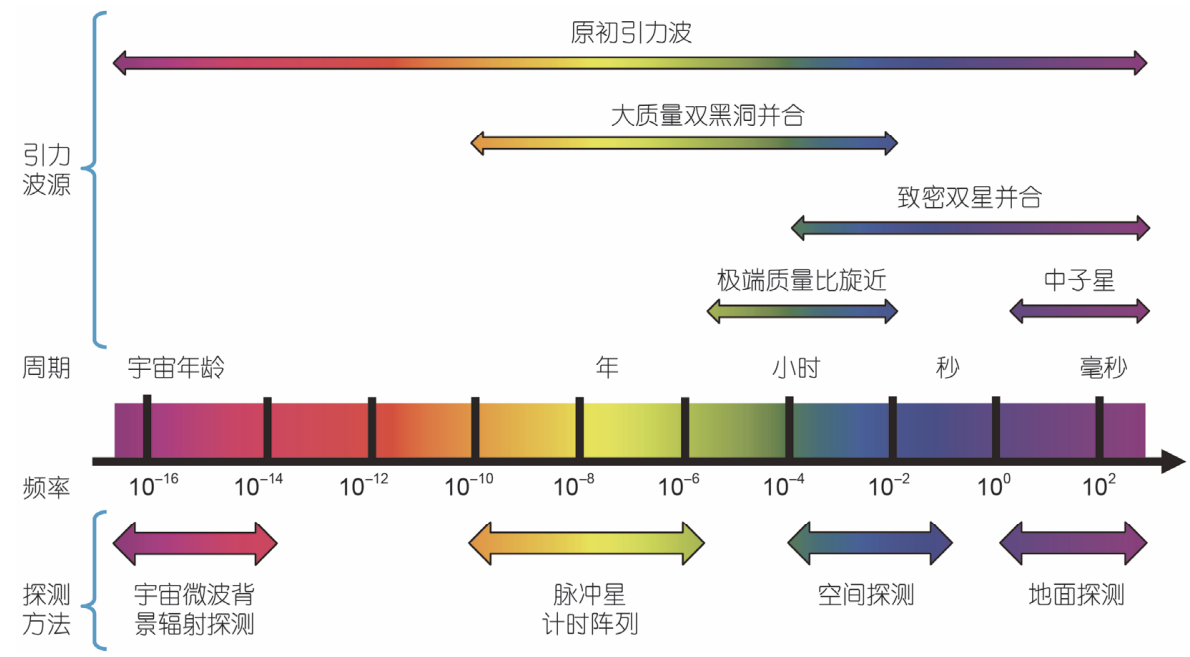

图 1 (网络版彩色)主要引力波源、频谱及探测方法

Figure 1 (Color online) Main gravitational wave sources, spectrum and detection methods 
器方案中, 卫星之间的距离可以从几千千米(如日本提出 的 DECIGO(DECi-hertz Interferometer Gravitational wave Observatory) ${ }^{[46]}$ )到几百万千米(如欧美提出的 LISA $^{[9]}$ ), 甚 至几亿千米(如我国提出的 ASTROD-GW(Astrodynamical Space Test of Relativity using Optical Devices optimized for Gravitational Wave detection $)^{[47]}$ ). 天琴将采用约 17 万千米 的星间距.

\section{3 天琴简介}

2006 年, 天琴计划团队研制的空间簧片加速度计成 功通过了搭载飞行, 各项指标均达到预期目标, 满足了用 户需求. 同时还开展了基于卫星的静电悬浮加速度计的飞 行验证. 2017 年 4 月, 团队设计研制的分辨率达到 $3 \times 10^{-10}$ $\mathrm{m} \mathrm{s}^{-2} \mathrm{~Hz}^{-1 / 2}$ 的高精度静电加速度计成功搭载, 在轨运行良 好. 同时, 团队在长距离激光干涉测量技术方面已有十多 年的研究积累. 在星载超稳激光干涉仪光学平台的研究上, 团队利用硅酸粘结技术已经成功制作出一体化高精度光 学干涉仪, 定位精度可达皮米量级. 在激光稳频的研究工 作中, 团队搭建星载激光稳频系统剩余频率噪声仅为 1.5 $\mathrm{Hz} \mathrm{Hz}{ }^{-1 / 2}$. 在高精度相位测量技术研究方面, 团队已经成 功研发完成基于锁相环原理的 FPGA 数字高精度相位计, 相位测量噪声本底达到 $10^{-6} \mathrm{rad} \mathrm{Hz}^{-1 / 2}$ 的水平. 在星间激光 干涉测量信号捕获跟踪技术方面, 团队搭建的模拟星间激 光干涉测量的干涉仪光路系统闭环控制时的角度控制精 度优于 $10^{-7} \mathrm{rad} \mathrm{Hz}{ }^{-1 / 2}$. 基于上述关键技术的进展, 团队于 2014 年正式提出天琴计划.

天琴将由 3 颗全同的卫星构成, 每颗卫星都在以地球 为中心、半径约 10 万千米的轨道上运行. 3 颗卫星构成一 个等边三角形编队, 约每 $3.6 \mathrm{~d}$ 绕地球转动一周. 天琴每年 跟随地球绕太阳一周, 运动过程中编队平面保持与黄道面
接近垂直, 并维持其法向的空间指向近似不变. 目前, 天 琴卫星编队平面的法向被选为指向一个特定的引力波源, RXJ0806.3+1527，使天琴对这个波源的引力波的响应最大 化, 用于对系统性能进行检验和标定, 为之后长期的天文 观测做准备 ${ }^{[17]}$. 目前也有建议将天琴的编队平面指向银心 方向. 天琴的初步概念方案如图 2 所示.

在关键技术方面，每颗天琴卫星内部都将包含由金、 铂等化学稳定、高密度金属制成的金属块(称检验质量), 它 们的作用是准确反映引力场的信息. 为此, 卫星平台需要 从动力学控制、温度控制、电磁兼容、结构设计、材料选 取等各个方面人手, 以保证为所有检验质量提供稳定安静 的工作环境, 抑制一切可能的非引力因素的干扰, 使得检 验质量处于自由落体状态下循测地线运动. 在此基础上, 还需要优化轨道设计, 使各个检验质量维持长期稳定的编 队飞行状态. 当有引力波通过时, 各检验质量之间的距离 会发生细微变化, 然后利用高精度激光干涉测量技术将这 些变化记录下来, 从而获得引力波信号的信息.

天琴初步方案要求: 在 $1 \mathrm{mHz} 1 \mathrm{~Hz}$ 的探测频段内, 约 17 万千米基线上的激光位移测量误差不超过 $10^{-12} \mathrm{~m} \mathrm{~Hz}^{-1 / 2}$ 量级, 检验质量的残余加速度不超过 $10^{-15} \mathrm{~m} \mathrm{~s}^{-2} \mathrm{~Hz}^{-1 / 2}$ 量 级. 在卫星平台方面, 卫星内部核心载荷区域的温度稳定性 达到 $10^{-4} \mathrm{~K}$ 量级, 部分核心区域达到 $10^{-6} \mathrm{~K}$ 量级, 用于卫星 无拖曳控制的微推进器的推力噪声应达到 $0.1 \mu \mathrm{N} \mathrm{Hz}{ }^{-1 / 2}$ 量 级、推力范围达到 1 100 $\mu \mathrm{N}$, 寿命应长达若干年 ${ }^{[17]}$. 天琴的 主要技术指标如表 1 所示.

\section{2 实施天琴计划的重要意义}

\section{1 科学意义}

引力波是人类观测宇宙的全新窗口. 天琴引力波天文

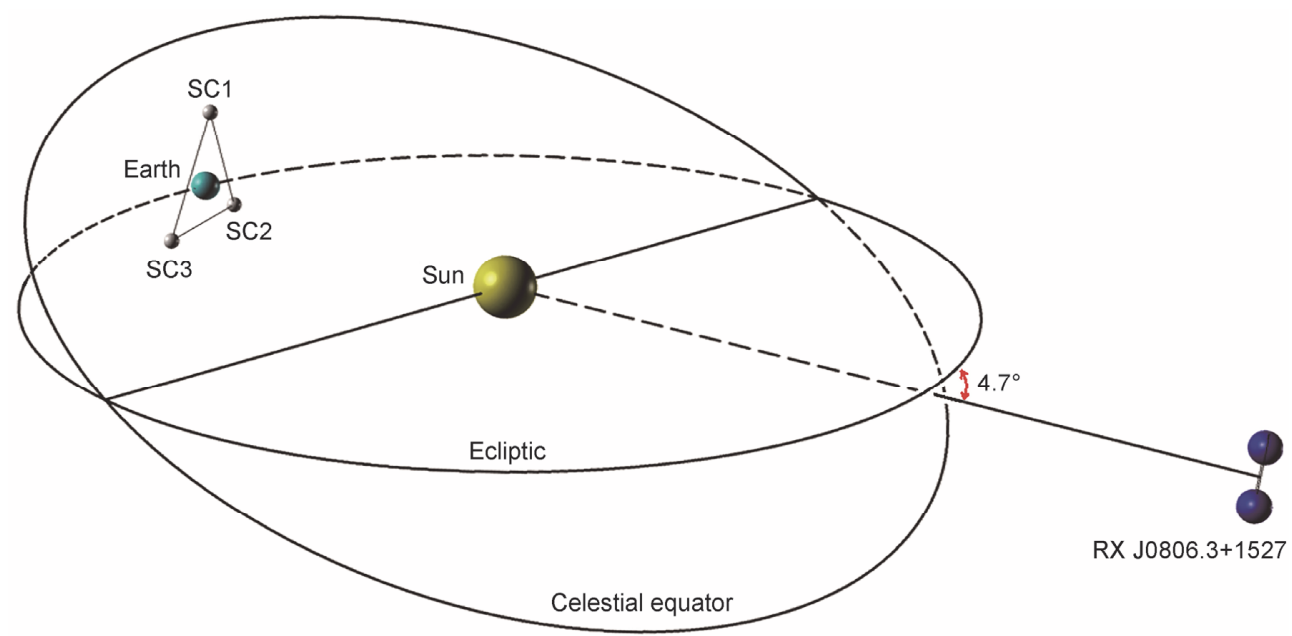

图 2 (网络版彩色) 天琴初步概念方案 ${ }^{[17]}$

Figure 2 (Color online) Preliminary concept scheme of TianQin ${ }^{[17]}$ 
表 1 天琴主要技术指标 ${ }^{[17]}$

Table 1 Main technical requirements of TianQin ${ }^{[17]}$

\begin{tabular}{ll}
\hline \multicolumn{1}{c}{ 参数 } & \multicolumn{1}{c}{ 取值 } \\
\hline 卫星数目 & $N=3$ \\
星座构型 & 等边三角形 \\
轨道类型 & 地心轨道 \\
臂长 & $L \sim 10^{5} \mathrm{~km}$ \\
位置测量精度 & $1 \mathrm{pm} \mathrm{Hz}^{-1 / 2} @ 6 \mathrm{mHz}$ \\
残余加速度精度 & $10^{-15} \mathrm{~m} \mathrm{~s}^{-2} \mathrm{~Hz}^{-1 / 2} @ 6 \mathrm{mHz}$ \\
观测窗口 & 每年 $2 \times(3$ 个月) \\
激光波长 & $\lambda=1064 \mathrm{~nm}$ \\
光功率 & $P$ opt $=4 \mathrm{~W}$ \\
望远镜口径 & $D=20 \mathrm{~cm}$ \\
\hline
\end{tabular}

台预期可以探测到种类多样的引力波源 (图 3), 可以帮助 研究致密双星系统的演化, 验证大质量黑洞的存在, 探索 黑洞和星系的形成, 研究宇宙的形成与演化, 在强场和极 端相对论条件下检验黑洞和引力本质等.

\section{1 .1 天琴与引力理论}

引力波是广义相对论的基本预言, 它提供了一个在极 端情况下检验广义相对论、研究引力的高阶非线性效应的 形态. 天琴引力波天文台预期测量到的引力波信号都源于 包含极强的引力场过程, 比如致密双星合并、两个超大质 量黑洞的合并、宇宙大爆炸等. 在这些预期可以被天琴探 测到的物理过程中, 引力的高阶非线性效应会有明显的体 现, 并最终在引力波的极化、相位和强度等信息上体现出 来. 天琴对引力高阶效应的探测可以帮助人们区分广义相 对论和其他可能的替代理论 ${ }^{[10,48]}$. 此外, 一些特定的引力 波信号, 如极端质量比旋近, 由于其在纯引力作用下作长 期复杂的演化, 因此被认为是绝佳的引力实验室 ${ }^{[49]}$.

天琴引力波天文台还可能帮助人们获得有关量子引

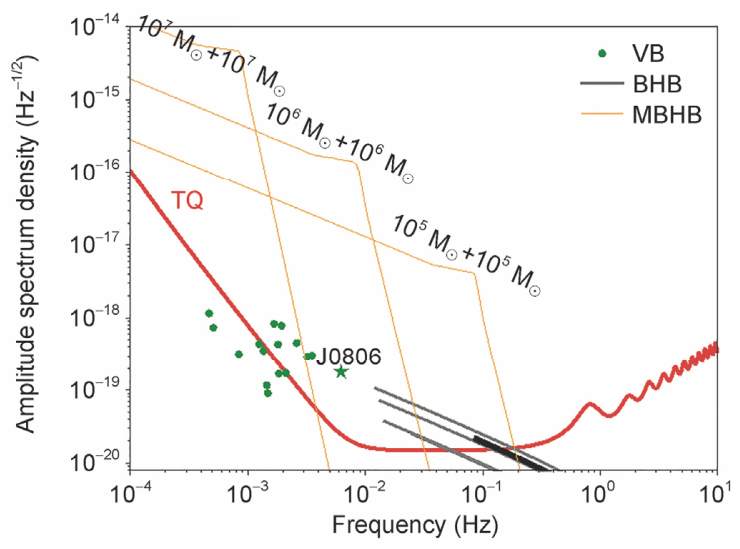

图 3 (网络版彩色)天琴灵敏度曲线以及各种主要引力波波源 Figure 3 (Color online) TianQin sensitivity curve and main gravitational wave sources
力的部分信息. 量子引力的特征能标 $\left(10^{19} \mathrm{GeV}\right)$ 远远超出 了目前高能粒子加速器所能达到的能量上限. 但是来自宇 宙大爆炸的原初引力波因为在极早期就与其他物质退耦, 因此有望携带有关引力量子特征的信息 ${ }^{[50]}$. 天琴引力波天 文台探测的频段范围对应宇宙在 $10^{-18} \sim 10^{-10} \mathrm{~s}$ 时退耦的引 力波, 对应当前高能粒子加速器所能达到的最高能量, 因 此天琴引力波探测有可能与地面的高能粒子实验形成相 互促进的关系.

\section{1 .2 天琴与天文学}

引力波将是除电磁波、中微子和宇宙射线之外可供人 们对宇宙作观测的新媒介. 作为一种新手段, 天琴引力波 天文台将极大地拓展引力波天文学这一全新的研究领域, 并有望极大促进对黑洞等极端致密天体及其前身星与其 周围环境共同演化的研究 ${ }^{[51]}$.

天琴引力波天文台可以通过对银河系内的致密双星 系统的观测, 测量其距离、轨道和质量等信息. 与此同时, 通过联合天琴与地面引力波探测器, 开展多波段引力波探 测, 可以帮助确定恒星级双黑洞的自旋、轨道偏心率等信 息. 这些信息对于研究双星演化和致密双星系统的形成机 制有着重要帮助 ${ }^{[52,53]}$.

天琴引力波天文台也可以对银河系外大质量黑洞进 行深人研究. 观测表明, 星系中心普遍存在大质量黑洞, 更进一步地，宿主星系与中心黑洞关系密切 ${ }^{[54]}$. 主流模型 认为, 星系的成长伴随着不断的星系并合, 而由于动力学 摩擦, 大质量黑洞也会随之靠近并最终并合 ${ }^{[55]}$. 然而, 星 系中心的大质量黑洞与宿主星系之间的联系、两者的共同 演化历史等, 当前还存在许多尚待解答的问题, 如大质量 黑洞的形成机制、大质量双黑洞如何在有限时长内耗散角 动量迅速并合等 ${ }^{[56]}$. 通过天琴对大质量双黑洞并合的引力 波探测, 有望给这类问题的解答带来启示.

\subsection{3 天琴与宇宙学}

天琴引力波天文台可以测量极早期宇宙的物理性质. 相比电磁波或中微子, 引力波在宇宙起源过程中更早地与 物质退耦, 因此来自宇宙大爆炸的原初引力波可以携带更 接近宇宙源点的信息. 原初引力波一方面可能在宇宙微波 背景辐射中留下可探测的印记; 另一方面, 原初引力波很 可能具有非热谱, 因此也可以被空间引力波实验探测到. 原初引力波的探测对于研究宇宙的演化和引力的量子特 性都将有极其重要的意义 ${ }^{[14,16]}$.

天琴引力波天文台同样可以对当下的宇宙学参数做 出限制. 通过两种电磁手段观测的宇宙学参数存在无法解 释的差异: 由宇宙微波背景辐射观测给定的哈勃常数与由 临近的 Ia 型超新星给定的哈勃常数无法吻合, 且其差异无 法用统计误差解释. 由于引力波观测可以直接测量光度距 离, 配合多信使探测的红移信息, 可以获得一个独立的宇 宙学参数测量 ${ }^{[57]}$. 由于可探测范围广阔, 天琴对这类标准 警笛的探测可以实现非常高精度的宇宙学参数测量 ${ }^{[58]}$. 这 
样一个独立、精确的测量将有望调和电磁波宇宙学观测中 的这一矛盾 ${ }^{[59 ~ 61]}$.

\section{2 战略价值}

天琴计划将极大推动高精度时空参考体系构建、超长 距离激光干涉测量、高精度卫星平台建设等一大批尖端空 间技术的发展，这些技术的发展对于国民经济和国家战略 需求都有重要的应用价值. 比如高精度星间激光测距技术 可以帮助实现高轨卫星精确编队飞行, 建立高精度时空坐 标体系 ${ }^{[62]}$; 高精度星载加速度计和星间激光干涉测量可以 用于下一代低低跟踪卫星重力测量, 进行高精度全球重力 场测绘 ${ }^{[63,64]}$; 高性能的卫星平台有望在未来空间科学实验 中发挥越来越重要的作用 ${ }^{[65]}$.

\section{3 天琴计划的规划与进展}

天琴计划的系统推进工作于 2015 年正式启动, 目前 在精密重力测量研究设施、载荷研究基地、激光测距台站、 空间引力波探测地面模拟装置、天琴计划系列试验卫星等 几个方面均取得重要的进展.

\section{1 精密重力测量研究设施}

精密重力测量研究设施由华中科技大学作为项目法 人, 中国科学院测量与地球物理研究所、中国科学院物理 与数学研究所、中国地质大学(武汉)和中山大学共建, 是 “十二五” 期间国家优先支持 16 项重大科技基础设施建设 项目之一. 精密重力测量研究设施以具备全球毫伽级、基 准微伽级的重力数据获取、评估与应用能力为目标, 主要 建设精密重力测量基准台与检测系统, 卫星、航空和水下 重力探测环境模拟与物理仿真试验系统, 以及全球高精度 重力场数据处理系统, 计划建成国际一流、综合指标国际 领先的精密重力测量研究设施, 并使之发展成为具有国际 影响力的重力测量科学中心之一, 为我国地球科学基础研 究及精密重力仪器研制、测量与应用研究提供必要的实验 条件. 精密重力测量研究设施的建设对于天琴计划的技术 积累和人才储备具有至关重要的意义. 项目建议书、可行 性研究报告以及初步设计概算分别于 2015 年 5 月 5 日、 2016 年 7 月 11 日、2018 年 11 月 16 日获得国家发展改革 委批复(发改高技[2015]947号, 发改高技[2016]1499号, 发 改投资[2018]1685 号), 这标志着精密重力测量国家重大科 技基础设施进人了建设启动阶段.

\section{2 载荷研究基地}

天琴计划载荷研究基地的主要目的是为空间引力波 探测各个核心问题的解决提供基本的实验室研究条件, 为 空间引力波探测关键技术与核心载荷的研发研制、为天琴 卫星平台关键技术攻关提供充分的实验平台. 天琴计划载
荷研究基地布局中山大学珠海校区, 建设内容包括面积约 3 万平方米的科研综合楼和 1 万平方米的山洞恒温实验室.

教育部于 2016 年批准筹建天琴计划教育部重点实验 室. 在珠海市政府大力支持下, 项目土建工程已于 2017 年 开工, 科研综合楼预计 2019 年交付使用.

\section{3 激光测距台站}

为了获得针对高轨卫星的激光测距能力, 天琴计划从 一开始就着手开展月球/高轨卫星激光测距研究, 主要包 括研制新一代激光测距角反射器, 以及建设地面激光测距 台站.

国家国防科工局探月与航天工程中心与国家自然科 学基金委于 2016 年立项支持开展月球中继星激光测距实 验. 在这个项目的支持下, 中国科学院云南天文台于 2018 年 1 月成功开展了国内首次月球激光测距实验. 目前, 中 山大学珠海校区正在新建配备 $1.2 \mathrm{~m}$ 望远镜的激光测距台 站, 将于 2019 年投人使用.

\section{4 空间引力波探测地面模拟装置}

空间引力波探测地面模拟装置的主要目的是针对空 间引力波探测在高精度空间惯性基准、长基线激光干涉测 量、高精度卫星平台、数值仿真与系统研究等方面的关键 技术问题，进行全系统的空间引力波探测过程模拟，为空 间引力波探测系统集成和整体性能评估提供完备的实验 平台.

空间引力波探测地面模拟装置布局于中山大学深圳 校区. 项目于 2016 年纳人《国家重大科技基础设施建设“十 三五”规划》备选项目序列，深圳市政府于 2018 年 6 月批 准了项目建议书.

\section{5 系列试验卫星}

天琴计划系列试验卫星将为核心关键技术提供地面 实验无法提供的在轨试验环境. 在天琴计划提出之初, 我 们制定了“0123”计划的技术路线图, 计划分步骤推进各方 面关键技术成熟. “0123”计划的主要内容包括:

步骤“0”: 开展月球/中继星激光测距实验, 发展超远 距离激光测距和高轨卫星精密定轨技术, 所取得的技术可 应用于等效原理检验等前沿引力物理研究. 这个项目需要 研制大孔径激光反射镜(封装是圆筒, 外形是圆), 因此称 为步骤“ 0 ”。

在国家国防科工局探月与航天工程中心与国家自然 科学基金委员会共同支持的月球中继星激光测距项目的 支持下，团队已经研制成功国际上精度最高的 $17 \mathrm{~cm}$ 口径 激光测距角反射器, 并已由嫦娥四号的鹊桥中继星于 2018 年 5 月 21 日搭载发射升空.

步骤“1”: 通过发射单颗卫星, 验证空间惯性传感、卫 星无拖曳控制等关键技术，所取得的关键技术可应用于空 
间等效原理检验等基础引力实验研究. 这个系列的项目需 要各发射单颗卫星, 因此称为步骤“ 1 ”. 中山大学、华中科技 大学、航天东方红卫星有限公司正在共同研制天琴计划的第 一颗技术试验卫星: 天琴一号. 目前该卫星项目已经由国家 国防科工局正式立项, 计划于 2019 年末发射进行实验.

步骤“2”: 通过部署两颗卫星, 验证高精度星间激光 测距技术, 所取得的关键技术可应用于下一代低低跟踪重 力场测量, 为对全球气候变化、水资源调查、地球物理学 等深人研究提供高精度全球重力数据. 这个系列的项目需 要各发射两颗卫星, 因此称为步骤“ 2 ”.

步骤“3”: 通过部署 3 颗卫星, 在太空中构建空间引力 波探测系统, 开展空间引力波探测研究. 这个系列的项目 需要各发射 3 颗卫星, 因此称为步骤“3”.

\section{4 国际合作}

可以预期, 到 21 世纪 30 年代, 世界上各国科学家通 力合作、利用多种手段联合对宇宙进行探测研究的大科学 合作将面临大发展. 通过与电磁波等其他天文观测手段联 合, 天琴可以开展多信使天文学研究; 通过与地面引力波 探测等其他波段的引力波探测手段联合, 天琴可以开展多 波段引力波天文学研究; 通过天琴计划发展起来的关键技 术, 还可以应用到地球物理学等其他学科领域. 因此, 开 展天琴计划国际合作, 建造和部署天琴空间引力波天文台, 通过广泛的国际合作开展科学探测研究, 不仅可以帮助加 深对宇宙和人类未来发展的认识, 也帮助加深对人类周边 生存环境的认识.

天琴计划自提出以来, 已经召开了多次国际研讨会, 得到了国际广泛关注和积极参与. 目前天琴合作组已经正 式成立, 来自俄罗斯、德国、意大利、英国、澳大利亚、 美国等在空间引力波探测领域有显著影响的著名科学家
加人了合作组咨询委员会. 天琴计划将坚持以我为主, 分 步推进的原则, 逐步与国外优势高校或科研组织建立课题 合作研究或全面合作协议等多方位、多层次合作关系, 积 极利用国外优质智力资源, 大力推进我国主导的空间引力 波探测事业.

\section{5 总结}

在各种主流的引力波探测手段中, 以天琴为代表的空 间引力波探测是能够对整个宇宙空间进行探测的独特方 式. 通过对大质量黑洞引力波辐射的研究, 空间引力波探 测有望对广义相对论检验给出最强的限制, 并为研究大质 量黑洞起源、星系核区动力学及演化等重大科学问题提供 无法通过其他手段获取的信息, 预期将在引力波天文学这 一新开辟的领域中发挥举足轻重的作用.

除了在科学上有重要意义, 空间引力波探测将引领高 精度时空参考体系构建、高精度星间激光干涉测量、超静 超稳卫星平台建设等一系列对国民经济和国家战略需求 有重要价值的前沿技术发展, 将在我国建设科技强国进程 中发挥显著的作用.

目前国际上所有的空间引力波探测计划都还处于设 计论证或预研究阶段. 2017 年 6 月, 欧空局正式选取 LISA 为其“宇宙憧憬 2015 2025”的 L3 项目，预期 2034 年发射， 这为我国在空间引力波探测领域积极开展研究工作、争取 抢占该领域的国际科技制高点提供了约 15 年的时间窗口. 通过及时部署、系统布局, 我国有希望在国际上率先实现 空间引力波探测.

空间引力波探测技术难度大、门槛高, 我国开展该项 目研究必然受到国际社会的广泛关注和欢迎. 天琴计划将 遵循“以我为主，积极发展对外合作”的原则，以天琴合作 组为依托, 共同实现引领世界的空间引力波探测的壮举.

\section{推苼阅读文献}

1 Abbott B P, Abbott R, Abbott T D, et al. Observation of gravitational waves from a binary black hole merger. Phys Rev Lett, 2016, 116: 061102

2 Abbott B P, Abbott R, Abbott T D, et al. GW151226: Observation of gravitational waves from a 22-solar-mass binary black hole coalescence. Phys Rev Lett, 2016 116: 241103

3 Abbott B P, Abbott R, Abbott T D, et al. GW170104: Observation of a 50-solar-mass binary black hole coalescence at redshift 0.2. Phys Rev Lett, 2017, 118: 221101

4 Abbott B P, Abbott R, Abbott T D, et al. GW170608: Observation of a 19 solar-mass binary black hole coalescence. Astrophys J, 2017, 851: L35

5 Abbott B P, Abbott R, Abbott T D, et al. GW170814: A three-detector observation of gravitational waves from a binary black hole coalescence. Phys Rev Lett, 2017, 119: 141101

6 LIGO Scientific Collaboration and Virgo Collaboration. GWTC-1: A Gravitational-Wave Transient Catalog of Compact Binary Mergers Observed by LIGO and Virgo during the First and Second Observing Runs. 2018, ArXiv: 1811.12907

7 Misner C W, Thorne K S, Wheeler J A. Gravitation. Princeton: Princeton University Press, 1973

8 Sathyaprakash B S, Schutz B F. Physics, astrophysics and cosmology with gravitational waves. Living Rev Relativ, 2009, 12: 2

9 Amaro-Seoane P, Audley H, Babak S, et al. Laser Interferometer Space Antenna. 2017, ArXiv: 1702.00786 
10 Berti E, Barausse E, Cardoso V, et al. Testing general relativity with present and future astrophysical observations. Class Quant Grav, 2015, 32: 243001

11 Hu Y M, Mei J, Luo J. Science prospects for space-borne gravitational-wave missions. Natl Sci Rev, 2017, 4: 683-684

12 Klein A, Barausse E, Sesana A, et al. Science with the space-based interferometer eLISA: Supermassive black hole binaries. Phys Rev D, 2016, 93: 024003

13 Caprini C, Hindmarsh M, Huber S, et al. Science with the space-based interferometer eLISA. II: Gravitational waves from cosmological phase transitions. J Cosmol Astropart Phys, 2016, (4): 1

14 Tamanini N, Caprini C, Barausse E, et al. Science with the space-based interferometer eLISA. III: Probing the expansion of the universe using gravitational wave standard sirens. J Cosmol Astropart Phys, 2016, (4): 2

15 Bartolo N, Caprini C, Domcke V, et al. Science with the space-based interferometer LISA. IV: Probing inflation with gravitational waves. J Cosmol Astropart Phys, 2016, (12): 26

16 Babak S, Gair J, Sesana A, et al. Science with the space-based interferometer LISA. V. Extreme mass-ratio inspirals. Phys Rev D, 2017, 95: 103012

17 Luo J, Chen L S, Duan H Z, et al. TianQin: A space-borne gravitational wave detector. Class Quant Grav, 2016, 33: 035010

18 Weber J. Gravitational radiation. Phys Rev Lett, 1967, 18: 498-501

19 Aasi J, Abbott B P, Abbott R, et al. Advanced LIGO. Class Quant Grav, 2015, 32: 074001

20 Acernese F, Agathos M, Agatsuma K, et al. Advanced Virgo: A second-generation interferometric gravitational wave detector. Class Quant Grav, 2015, 32: 024001

21 Aso Y, Michimura Y, Somiya K, et al. Interferometer design of the KAGRA gravitational wave detector. Phys Rev D, 2013, 88: 043007

22 Luck H, Affeldt C, Degallaix J, et al. The upgrade of GEO600. J Phys Conf Ser, 2010, 228: 012012

23 Iyer B, Souradeep T, Unnikrishnan C S, et al. LIGO-India, Proposal of the Consortium for Indian Initiative in Gravitational-wave Observations (indigo). Technical Report LIGO-M1100296, LIGO-India, November 2011

24 Manchester R N, Hobbs G, Bailes M, et al. The Parkes Pulsar Timing Array Project. Publ Astron Soc Aust, 2013, 30 : e017

25 Desvignes G, Caballero R N, Lentati L, et al. High-precision timing of 42 millisecond pulsars with the European Pulsar Timing Array. Mon Not Roy Astron Soc, 2016, 458: 3341-3380

26 Arzoumanian Z, Brazier A, Burke-Spolaor S, et al. The NANOGrav 11-year data set: High-precision timing of 45 millisecond pulsars. Astrophys J Suppl Ser, 2018, 235: 37

27 Nan R, Li D, Jin C, et al. The Five-hundred-meter Aperture Spherical Radio Telescope (FAST) project. Int J Mod Phys D, 2011, 20: 989-1024

28 Spergel D N, Verde L, Peiris H V, et al. First-year Wilkinson Microwave Anisotropy Probe (WMAP) observations: Determination of cosmological parameters. Astrophys J Suppl S, 2003, 148: 175-194

29 European Space Agency. Planck: The Scientific Programme. 2006, ArXiv: astro-ph/0604069

30 Ade P, Aikin R W, Barkats D, et al. Detection of B-mode polarization at degree angular scales by BICEP2. Phys Rev Lett, 2014, 112: $1-26$

31 Li H, Li S Y, Liu Y, et al. Probing primordial gravitational waves: Ali CMB polarization telescope. Natl Sci Rev, 2019, 6: 145-154

32 Abbott B P, Abbott R, Abbott T D, et al. Exploring the sensitivity of next generation gravitational wave detectors. Class Quant Grav, 2017, 34: 044001

33 Punturo M, Abernathy M, Acernese F, et al. The Einstein Telescope: A third-generation gravitational wave observatory. Class Quant Grav, 2010, 27: 194002

34 LIGO Scientific Collaboration. Instrument Science White Paper. Technical Report, LIGO Scientific Collaboration. 2015

35 Miao H, Ma Y, Zhao C, et al. Enhancing the bandwidth of gravitational-wave detectors with unstable optomechanical filters. Phys Rev Lett, 2015, 115: 211104

36 Decher R, Randall J L, Bender P L, et al. Design aspects of a laser gravitational wave detector in space. Active optical devices and applications. Proc SPIE, 1980, 149-153

37 Faller J E, Bender P L, Hall J L, et al. Space antenna for gravitational wave astronomy. Kilometric Optical Arrays in Space, ESA Special Publication, 1985

38 NASA. Physics of the Cosmos Gravitational-Wave Mission Concept Study Final Report. Technical Report, 2012

39 Harry G M, Fritschel P, Shaddock D A, et al. Laser interferometry for the Big Bang Observer. Class Quant Grav, 2006, 23: 7361

40 Isoyama S, Nakano H, Nakamura T. Multiband gravitational-wave astronomy: Observing binary inspirals with a decihertz detector, B-DECIGO. Prog Theor Exp Phys, 2018, 2018: 073E01

41 LISA Study Team. LISA Pre-phase A Report. 2nd ed. Technical Report, MPQ. 1998 
42 Amaro-Seoane P, Aoudia S, Babak S, et al. eLISA: Astrophysics and cosmology in the millihertz regime. GW Notes, 2013, 6: 4-110

43 Armano M, Audley H, Auger G, et al. Sub-Femto- $g$ free fall for space-based gravitational wave observatories: LISA pathfinder results. Phys Rev Lett, 2016, 116: 231101

44 Armano M, Audley H, Auger G, et al. Charge-induced force noise on free-falling test masses: Results from LISA pathfinder. Phys Rev Lett, 2017, 118: 171101

45 Armano M, Audley H, Baird J, et al. Beyond the required LISA free-fall performance: New LISA pathfinder results down to $20 \mu \mathrm{Hz}$. Phys Rev Lett, 2018, 120: 061101

46 Kawamura S, Ando M, Nakamura T, et al. The Japanese space gravitational wave antenna-DECIGO. J Phys Conf Ser, 2008, $122: 012006$

47 Ni W T. Astrod-GW: Overview and progress. Int J Mod Phys D, 2013, 22: 1341004

48 Berti E, Buonanno A, Will C M. Testing general relativity and probing the merger history of massive black holes with LISA. Class Quant Grav, 2005, 22: S943-S954

49 Gair J R, Vallisneri M, Larson S L, et al. Testing general relativity with low-frequency, space-based gravitational-wave detectors. Living Rev Relat, 2013, 16: 7

50 eLISA Consortium, Amaro-Seoane P, Aoudia S, et al. The Gravitational Universe. 2013, ArXiv: https://arxiv.org/abs/1305.5720

51 LIGO Scientific Collaboration and Virgo Collaboration. Multimessenger Astronomy. 2011, ArXiv: 1105.5843

52 Breivik K, Rodriguez C L, Larson S L, et al. Distinguishing between formation channels for binary black holes with LISA. Astrophys J, 2016, 830: L18

53 Nishizawa A, Berti E, Klein A, et al. eLISA eccentricity measurements as tracers of binary black hole formation. Phys Rev D, 2016, 94: 064020

54 Mo H, van den Bosch F C, White S. Galaxy Formation and Evolution. Cambridge: Cambridge University Press, 2010

55 Milosavljevic M, Merritt D. The final PARSEC problem. Proceeding of the Astrophysics of Gravitational Wave Sources for American Institute of Physics Conference Series, 2003. 201-210

56 Salcido J, Bower R G, Theuns T, et al. Music from the heavens-gravitational waves from supermassive black hole mergers in the EAGLE simulations. Mon Not Roy Astron Soc, 2016, 463: 870-885

57 Schutz B F. Determining the Hubble constant from gravitational wave observations. Nature, 1986, 323: 310-311

58 Holz D E, Hughes S A. Using gravitational-wave standard sirens. Astrophys J, 2005, 629: 15-22

59 Freedman W L. Cosmology at a crossroads. Nat Astron, 2017, 1: 0121

60 Chen H Y, Fishbach M, Holz D E. A two per cent Hubble constant measurement from standard sirens within five years. Nature, 2018, 562: 545-547

61 Abbott B P, Abbott R, Abbott T D, et al. A gravitational-wave standard siren measurement of the Hubble constant. Nature, 2017, 551: $85-88$

62 ESA. Laser Interferometer Space Antenna: A Cornerstone Mission for the Observation of Gravitational Waves. Technical Report, ESA-SCI, ESA. 2000

63 Li Q, Xue C, Liu J P, et al. Measurements of the gravitational constant using two independent methods. Nature, 2018, 560: 582-588

64 Sheard B S, Heinzel G, Danzmann K, et al. Intersatellite laser ranging instrument for the GRACE follow-on mission. J Geod, 2012, 86: 1083-1095

65 ESA. LISA-unveiling a Hidden Universe: Assessment Study Report. Technicalw Report, ESA/SRE, ESA. 2011 


\title{
TianQin project and international collaboration
}

\author{
Yiming $\mathrm{Hu}^{1,2}$, Jianwei $\mathrm{Mei}^{1,2} \&$ Jun Luo ${ }^{1,2^{*}}$ \\ ${ }^{1}$ TianQin Research Center for Gravitational Physics, Sun Yat-sen University, Zhuhai 519082, China; \\ ${ }^{2}$ School of Physics and Astronomy, Sun Yat-sen University, Zhuhai 519082, China \\ *Corresponding author, E-mail: junluo@mail.sysu.edu.cn
}

The proposed TianQin gravitational wave observatory is aiming to detect gravitational wave signals in the millihertz frequency band, through the method of space-borne laser interferometry. In total, three satellites will be launched, each following a geocentric orbit, with an orbital altitude of about one hundred thousand kilometers. They form into an equilateral triangle formation, with an orbital period of $3.6 \mathrm{~d}$. The orbital plane of TianQin is fixed almost constant, towards one specific gravitational wave source, RXJ0806.3+1527, so that TianQin's response to this source is maximized, and this source can be used to calibrate and assess the facilities performance.

It is widely anticipated that in the millihertz frequency band, there lurks a wide variety of gravitational wave sources, ranging from massive black hole binary mergers, extreme mass ratio inspirals, Galactic binary white dwarves, stellar mass binary compact objects inspirals, as well as stochastic gravitational wave background. Meanwhile, the expected source number in such frequency band would also be huge. The successful implementation of the TianQin project would pave the way towards exciting prospects of future gravitational wave research. By successfully performing gravitational wave observation, the TianQin gravitational wave observatory can open a new window for the study of gravitational wave astronomy, gravitational wave cosmology as well as gravitational wave physics. The science prospect of TianQin gravitational wave observation can be further enhanced through a combination with multi-band gravitational wave detection and multi-messenger astronomical observations, by collaboration with gravitational wave detectors operating in different frequency bands as well as with other probes focusing on different messengers like electro-magnetic signals, neutrinos or cosmic rays. The TianQin project will also greatly promote the construction of a high-precision space-time reference system, ultra-long-range laser interferometry, high-precision satellite platform, etc. The development of cutting-edge space technologies has important applications in the national economy as well as the national strategic needs.

Currently, the TianQin project has made important progress in the precision gravity measurement facility, the payload research base, the laser ranging station, the simulation facility for space-borne gravitation wave detection on ground, and the series test satellite for the TianQin project. Based on the long-term accumulation of relevant technology, the TianQin project team proposed the goal of achieving gravitational wave detection in the 2030s, and a "0123" plan roadmap is formulated, aiming to perform, step by step, lunar or relay satellite laser ranging, testing key technology like space-borne laser interferometry and drag-free control, verifying high precision space-borne laser interferometry technology, and ultimately detecting gravitational wave.

By the 2030s, the scientific cooperation between scientists from all over the world and the use of various means to jointly conduct exploration and research on the Universe will face great development. So far, the TianQin project has held many international seminars and received extensive international attention and active participation. Currently, the TianQin Collaboration Group has been formally established, with a collaboration group advisory committee also established, consisting of scientists with significant influence in the field of gravitational wave detection all over the world. The TianQin project will establish a collaborative research or comprehensive cooperation agreement with foreign universities or research organizations, and vigorously promote the China-led space-borne gravitational wave detection business. Through extensive international cooperation, the TianQin project will unite domestic and foreign strengths and strive to take the lead in realizing the gravitational wave detection in the window of millihertz band.

\section{TianQin, gravitational wave, international collaboration, space-borne gravitational wave detection}

doi: 10.1360/N972019-00046 